\title{
Hallazgos inmunohistoquímicos en una muestra de pacientes con cáncer de mama atendidos del 2009 al 2013 en el Hospital Ángeles Pedregal
}

\author{
Consuelo Hernández Álvarez, ${ }^{1}$ Catalina Romo Aguirre, ${ }^{2}$ Jean René V Clemenceau, ${ }^{3}$ \\ Jorge Valenzuela Tamariz, ${ }^{4}$ Blanca Fátima Ayala Pacheco ${ }^{1}$
}

\section{Resumen}

Introducción: El cáncer de mama es la primera causa de muerte en la mujer en México. Es una enfermedad heterogénea que demanda un abordaje multidisciplinario, con el sustento tecnológico adecuado a fin de implementar mejores estrategias terapéuticas. Con base en su clasificación y grado de malignidad, se debe definir el pronóstico y tratamiento, lo que a su vez logrará favorecer la calidad de vida y la supervivencia. Objetivo: Determinar las características inmunohistoquímicas de una muestra de pacientes con diagnóstico de cáncer de mama que acudieron al Hospital Ángeles Pedregal del 2009 al 2013. Material y métodos: Estudio descriptivo, retrolectivo, longitudinal, en el cual se analizaron expedientes clínicos y resultados de histopatología; la muestra total fue de 417 pacientes durante el periodo de enero de 2009 a diciembre de 2013. Resultados: Se analizaron 417 casos, de los cuales el principal tipo histopatológico encontrado fue: ductal infiltrante sin patrón específico $(71.94 \%)$. El tratamiento quirúrgico conservador se realizó en $25 \%$ mientras que el radical en $37 \%$. Conclusiones: Este estudio mostró una diferencia en la edad media de diagnóstico $\dot{X}$ (56 años \pm $13)$, en comparación con el promedio nacional de 60 . La mortalidad fue de $1.9 \%$, por debajo del promedio nacional.

Palabras clave: Cáncer de mama, inmunohistoquímica, histopatológico.

\section{Summary}

Introduction: Breast cancer is the leading cause of death in women in Mexico, and this is a heterogeneous disease, which requires a multidisciplinary approach, with the appropriate technological support to implement therapeutic strategies based on their classification and degree of malignancy which could inference of the prognosis and treatment, as well as in the patients with advanced disease could promote the quality of life and survival. Objective: Determine the characteristics, surgical, histopathology and immunohistochemical of patients with a diagnosis of Breast Cancer at the Hospital Ángeles Pedregal. Material and methods: We realize a retrospective, descriptive, longitudinal study, where 417 clinical records and histopathological reports were analyzed during the period from January 2009 to December 2013. Results: We obtained from the 417 cases that the main histopathological type was the ductal infiltrating carcinoma with $71.94 \%$. Surgical conservative treatment was conducted in a $25 \%$ while radical treatment was made in $37 \%$. The Mortality rate was $1.9 \%$ which results below the national average. Conclusions: It is essential to recognize that the morbidity of this pathology is duly detectable and close monitoring is essential to improve the quality of life of the patients.

Key words: Breast cancer, inmunohistochemical, histopathology.

\footnotetext{
1 Residente de Ginecología y Obstetricia del Hospital Ángeles Pedregal, Facultad Mexicana de Medicina de la Universidad La Salle.

2 Médico de Anatomía Patológica del Hospital Ángeles Pedregal.

3 Centro de Cáncer del Hospital Ángeles Pedregal.

4 Jefe del Departamento de Patología del Hospital Ángeles Pedregal.
}

Correspondencia:

Dra. Consuelo Hernández Álvarez

Correo electrónico: cheloah83@hotmail.com

Aceptado: 02-01-2017.

Este artículo puede ser consultado en versión completa en http://www.medigraphic.com/actamedica 


\section{INTRODUCCIÓN}

En México, el cáncer de mama ocupa el primer lugar en incidencia de las neoplasias malignas en las mujeres. Representa el $11.34 \%$ de todos los casos de cáncer. Existe un incremento global de aproximadamente 1.5\% anual; sin embargo, en los países de economía emergente este incremento es de alrededor de un 5\%. El grupo de edad más afectado se encuentra entre los 40 y los 59 años. ${ }^{1}$ La mortalidad por cáncer mamario se ha incrementado en un $10.9 \%$ relativo durante los últimos años. En 2006, el cáncer de mama fue la segunda causa de muerte entre mujeres de 30 a 54 años y la tercera con más frecuencia entre el grupo de 30 a 59 años. ${ }^{9}$

El cáncer de mama, en etapas iniciales, se presenta de manera subclínica en la mayoría de los casos, es decir, que solamente se detecta por estudios de imagen (mastografía, ultrasonido y resonancia magnética); en menor proporción por clínica (tumores palpables). Sin embargo, otra forma de presentación común es como un tumor no doloroso que hasta en $30 \%$ se asocia con adenopatías axilares.

Los tumores localmente avanzados en nuestro país representan el $70 \%$ de las etapas clínicas al momento del diagnóstico y pueden incluir cambios cutáneos como edema, ulceración, así como afectación de ganglios supra- e infraclaviculares homolaterales. ${ }^{2,3}$

En nuestro país, entre el 50 y $60 \%$ de todos los casos son detectados en etapas avanzadas, y únicamente el $22 \%$ de las mujeres reportan haber practicado un estudio diagnóstico en el año previo, esto debido a la influencia de múltiples factores socioculturales.

La mastografía es, hasta ahora, el mejor método de detección. Tiene una sensibilidad diagnóstica de 70 a $85 \%$, aunque 10 a $15 \%$ de los tumores pueden permanecer ocultos, sobre todo en mujeres con mamas densas. El ultrasonido es, en algunos casos, una herramienta complementaria para diferenciar masas quísticas de sólidas, para caracterizar lesiones benignas y malignas, y como guía para la realización de biopsias de lesiones no palpables. ${ }^{9}$

El procedimiento de elección es la toma de biopsias con aguja de corte $\left(\operatorname{Trucut}^{\circledR}\right)$, tanto en lesiones palpables como en las no palpables. Esta forma diagnóstica se asocia con una exactitud de hasta el 98.5\%. ${ }^{4}$ En lesiones no palpables la biopsia debe ser realizada bajo la guía de algún método de imagen (ultrasonido, mastografía, resonancia). ${ }^{4}$

La evaluación patológica del cáncer de mama debe incluir, de manera indispensable, tipo histológico, grado nuclear, permeación vascular y linfática, tamaño del tumor, tipo de márgenes, número de ganglios linfáticos afectados y tamaño de la metástasis ganglionar, así como estudios de inmunohistoquímica que evalúen la presencia o no de receptores hormonales para estrógenos, progesterona, Ki67 y la sobreexpresión del gen ErbB2 (Her2/neu), además de estudios complementarios como citoqueratinas y factores de crecimiento epidérmico. ${ }^{5}$

Considerando que las características epidemiológicas de las pacientes atendidas en esta unidad hospitalaria deberían ser concordantes con la estadística nacional, se evaluaron las características inmunohistoquímicas de una muestra de pacientes que acudió para ser atendida por cáncer de mama, así como variables demográficas que pudieron recabarse de su expediente, esto con el fin de conocer la población que acude al hospital y proponer en un futuro medidas para protocolizar el manejo e implementar herramientas de prevención.

\section{MATERIAL Y MÉTODOS}

Se realizó un estudio descriptivo, retrolectivo, longitudinal, en donde se analizaron las historias clínicas y estudios histopatológicos de una muestra de 417 pacientes que fueron tratadas en el Hospital Ángeles Pedregal de enero del 2009 a diciembre del 2013 con diagnóstico corroborado de cáncer de mama.

Se estudiaron las siguientes características del tumor: su lateralidad, ubicación y tamaño, así como los resultados de biopsia, ganglio centinela y disección axilar, además del tipo de procedimiento quirúrgico realizado, ya sea cirugía conservadora o cirugía radical.

En cuanto a los reportes histopatológicos, se recabaron datos donde se describieron el tamaño del tumor, el tipo histológico y grado histológico de acuerdo con la clasificación de Nottingham y la presencia de cáncer in situ utilizando el índice pronóstico de Van Nuys. ${ }^{8}$

Los resultados de los estudios de inmunohistoquímica se realizaron obteniéndose el porcentaje de expresión de receptores a: estrógenos, progesterona, p53 y Ki-67, además de la presencia de Herb-2/neu y el subtipo molecular correspondiente.

\section{RESULTADOS}

Para el análisis estadístico se realizó inicialmente una descripción de la muestra y las variables de estudio con estadística descriptiva. Además se efectuó un análisis de asociación con $\chi^{2}$ para las variables nominales y una correlación de Pearson para las variables ordinales y cuantitativas.

Se realizó una comparación entre: grupos para el tipo de tumor y los resultados de las pruebas inmunohistoquímicas y las pacientes que fueron tratadas con una resección conservadora versus una resección radical.

En la muestra de estudio $(n=417)$, se presentaron 415 mujeres y 2 hombres; la edad de los pacientes promedio fue de 55 años (DE \pm 13 ) (Figura 1). 
Figura 1. Distribución por grupos de edad para pacientes con diagnóstico de cáncer de mama $\dot{X}=55, D E \pm 13$, rango $24-97$ años en comparación con datos de INEGI 2010.

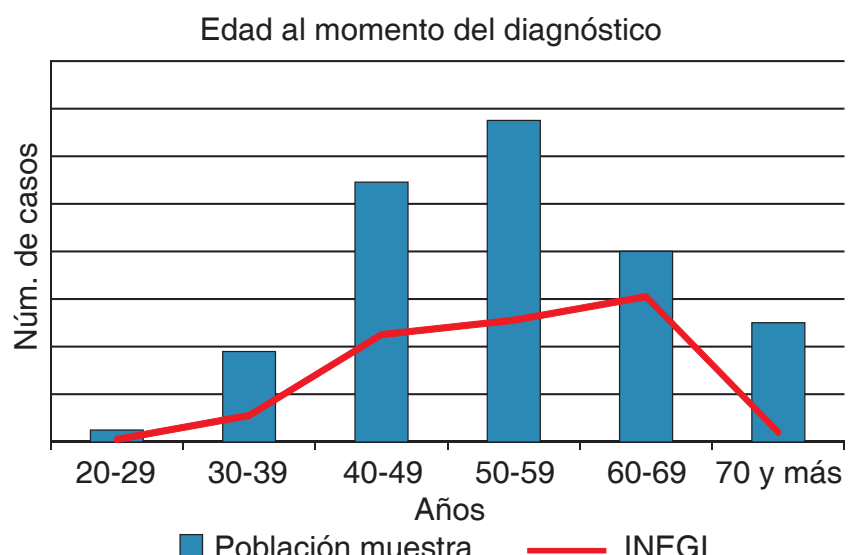

\begin{tabular}{|cc}
\hline \multicolumn{2}{|c}{$\begin{array}{c}\text { Cuadro 1. Características demográficas de la } \\
\text { población en estudio. }\end{array}$} \\
\hline Variable & Promedio \\
\hline Total pacientes & 417 \\
Femenino & 415 \\
Masculino & 2 \\
Edad promedio & 55 años $( \pm 13)$ \\
Lateralidad & $210(50.35 \%)$ \\
Derecho & $191(45.56 \%)$ \\
Izquierdo & $2(0.47 \%)$ \\
Bilateral & $14(3.35 \%)$ \\
Desconocido &
\end{tabular}

Lateralidad del cáncer: mama derecha en 50.35\% (n $=210$ ), mama izquierda en el $45.56 \%$ (191); origen desconocido en $3.35 \%$ (14); en dos casos fueron bilaterales $(0.47 \%)$ (Cuadro 1$)$.

El estudio de histopatología reportó mayor porcentaje de carcinoma ductal infiltrante con un $71.94 \%$ y carcinoma lobulillar en un 11\%. De los carcinomas in situ se encontró una frecuencia de: ductal in situ $11.27 \%$ y lobulillar in situ 3.14\%; otros tipos de cáncer se presentaron con un $2.63 \%$, como son el medular, papilar, cribiforme y mucinoso (Figura 2).

El tratamiento usado en mayor medida fue la cirugía radical en 155 (37\%) pacientes, cirugía conservadora en 105 (25\%). La cirugía con resección radical se asoció de manera significativa con el tamaño del tumor $\left(\chi^{2}=71.27\right.$, $p=.001)$; el resto de las variables no se asoció con el tipo de tratamiento.
Figura 2. Tipo histopatológico.

(DCIS: ductal in situ, LCIS: Iobulillar in situ, Otros: medular, papilar, mucinoso, cribiforme).

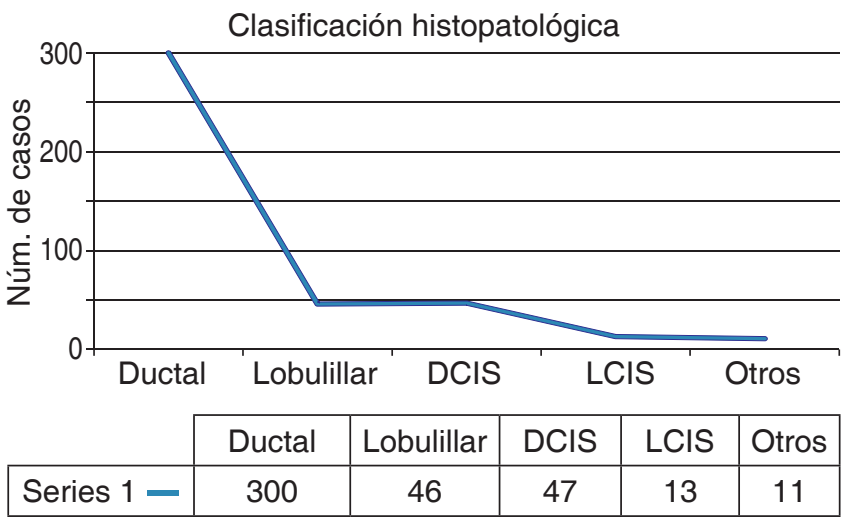

En relación al estudio histopatológico se tomaron biopsias en 151 (36\%), además de hacerse revisión de laminillas de mastectomías en seis casos, lo que corresponde a un $2 \%$.

Analizando los resultados de los estudios de inmunohistoquímica se encontró que este procedimiento se efectuó en 324 de los pacientes, lo que corresponde al $77.69 \%$ y de éstos se extrajeron los siguientes resultados: receptores estrógeno positivos, 271 (64.98\%); progesterona, 250 (59.95\%), y Herb2/neu positivo, $76(1.22 \%$ ) casos, mientras que fue negativo en 248 (59.47\%) pacientes (Figura 3).

En cuanto al tipo histológico y la expresión inmunohistoquímica se encontró que el tipo ductal presentaba diferencias en los receptores a estrógenos, comparado con el resto de tipos histológicos ( $F=4.917, p=.001)$. El tipo lobulillar presentaba mayores niveles de receptores a progesterona $(F=3.11, p=.015)$. La proteína $p-53$ no mostró diferencias significativas en el porcentaje de expresión por tipo histológico. La proteína ki67 se expresó en mayores porcentajes en el tipo ductal, comparado con el resto de tipos histológicos. ( $\mathrm{F}=6.54, \mathrm{p} \leq$.005) (Cuadro 2).

\section{DISCUSIÓN}

Actualmente se vive una transición epidemiológica tanto de las enfermedades como de su mortalidad y sin lugar a dudas la inclusión del cáncer como una de las principales causas representa un reto de alta importancia para la población y los sistemas de salud, pues los costos derivados de la atención oncológica se han incrementado de forma sostenida a lo largo de las últimas décadas y se debe considerar que en un futuro serán económicamente insostenibles, por lo que se debe maximizar la administración de los recursos. 
Las diferencias en la atención médica de este problema y sus consecuencias e impacto en la salud, se reflejan en las estadísticas de años de vida perdidos por muerte prematura y pérdida en la calidad de vida por discapacidad, lo que justifica llevar a cabo acciones específicas y sistematizadas para el diagnóstico temprano y tratamiento oportuno, contribuyendo a mejorar la vida de quienes padecen esta patología. ${ }^{10}$

En cuanto al cáncer de mama y su comportamiento epidemiológico, en la República Mexicana se ha registrado un incremento en su incidencia. En el año 2006 se constituyó como segunda causa de muerte en mujeres de 30 a 54 años, con una media de $26.1 \%$ x 100,000 en 2009, siendo actualmente la primera causa de muerte en mujeres de 30 a 59 años, ${ }^{1}$ secundario a factores de riesgo como el sobrepeso, baja lactancia materna, poca actividad física

Figura 3. Resultado inmunohistoquímico de anticuerpos.

RE: receptores estrógeno y RP: receptor progesterona (factores de transcripción clasificados de acuerdo al score de Allred), Ki67 (marcador de proliferación celular), Herb2/neu (proto-oncogén de la familia del receptor de crecimiento epidérmico y tirosina quinasa).

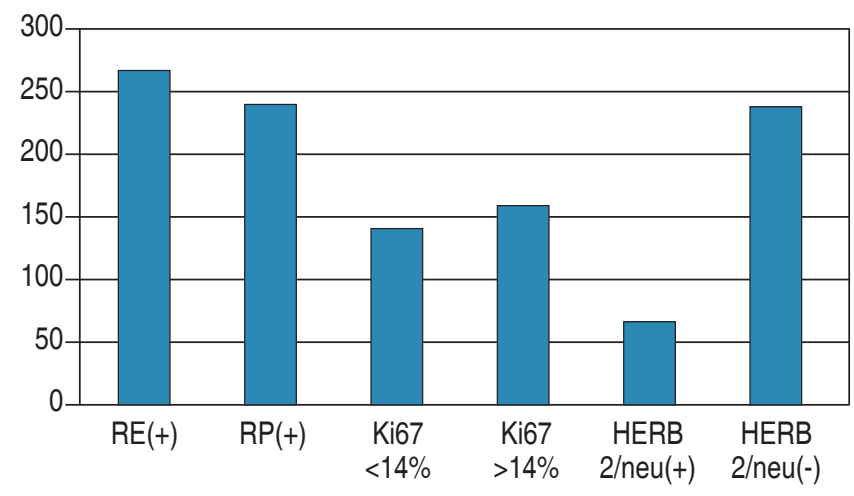

y exposición a hormonas, además de que se diagnostica tardíamente con alta mortalidad. ${ }^{11}$

De continuar con esta tendencia, en las próximas décadas observaremos un incremento significativo, tanto de la incidencia como de la prevalencia y la mortalidad, con un incremento global de al menos $1.5 \%$ anual. ${ }^{1}$ Esto nos obliga a replantear el protocolo y las guías de atención que se están llevando, haciendo mayor énfasis en la detección, especialmente en pacientes con factores de riesgo, con objeto de mejorar tanto la calidad de vida como la supervivencia.

Nuestros resultados son similares a los descritos en la literatura mundial sobre esta patología; al analizar los datos recopilados, podemos inferir que la mediana de edad al momento del diagnóstico es aproximadamente cinco años menor en nuestra población en comparación con otros países, y que a su vez la mayor parte de los casos se diagnostica en etapas tardías. En este aspecto debemos considerar que las variables demográficas en población latinoamericana no son las mismas que en la población mundial. Al analizar los estudios mexicanos encontramos que los resultados de nuestro estudio son muy semejantes a otros dos realizados con metodología similar. En uno realizado en Monterrey dentro del Instituto Mexicano de Seguridad Social con una muestra de 3,488 pacientes se encontró que la incidencia de cáncer de mama aumentó de 26.7 a 49.8 por 100,000 entre 2003 y 2011; la edad media fue de 55.7 y se mantuvo estable a través del tiempo; el principal tipo histológico que predominó fue el adenocarcinoma ductal infiltrante; el $42.5 \%$ tuvo ganglio y en relación a receptores de estrógenos, $64 \%$ fue RE +. El tipo histológico se asoció con edad mayor a 60 años, así como en casos positivos a receptores hormonales. ${ }^{11}$ Esto también se replicó en una muestra del Instituto Nacional de Cancerología que evaluó retrospectivamente la población atendida del 2007 al 2013, con una muestra de 3,109 casos y un promedio de edad

\begin{tabular}{|c|c|c|c|c|c|}
\hline \multicolumn{6}{|c|}{ Cuadro 2. Expresión de receptores por inmunohistoquímica. } \\
\hline Tipo & RE & $\mathrm{RP}$ & $P-53$ & Ki67 & $\mathrm{p}$ \\
\hline Ductal infiltrante & $61.67 \pm 45.5$ & $41.73 \pm 41.83$ & $17.73 \pm 32.62$ & $\begin{array}{c}29.05 \pm 29.03 \\
p \leq .005\end{array}$ & $p=.001$ \\
\hline Lobulillar & $82.19 \pm 37.14$ & $60.89 \pm 43.23$ & $14.80 \pm 26.99$ & $18.93 \pm 12.71$ & $p=.015$ \\
\hline DCIS & $53.38 \pm 46.87$ & $36.36 \pm 40.5$ & $25.29 \pm 33.73$ & $16.04 \pm 15.28$ & \\
\hline LCIS & $32.84 \pm 46.59$ & $29 \pm 42.47$ & $5.15 \pm 7.82$ & $4.38 \pm 2.59$ & \\
\hline Otros & $37.45 \pm 49.60$ & $29.27 \pm 41.36$ & $22 \pm 36.89$ & $28.81 \pm 31.82$ & \\
\hline Total de la muestra & $61.46 \pm 45.89$ & $42.51 \pm 42.27$ & $17.98 \pm 31.88$ & $25.69 \pm 22.83$ & \\
\hline
\end{tabular}


de 50 años, del cual 22\% expresaron HER2, con alto índice de masa corporal, en etapas mayores de IIA en el 70\% de los casos y el $84 \%$ a carcinoma ductal. ${ }^{12}$ Llama la atención el que, a pesar de que son poblaciones con características socioeconómicas diferentes a la de nuestro centro de salud, no se asocia una detección en etapas más tempranas. Las características histológicas fueron las mismas y también el tipo inmunohistoquímico encontrado.

Se observa que en esta unidad hospitalaria se diagnosticaron y trataron en promedio 84 nuevos casos al año, en los cuales la prevalencia de sobrepeso y obesidad en las pacientes con cáncer de mama es mayor al 70\%, por lo que debe ser una variable a evaluar en investigaciones posteriores y que ya se ha referido como factor de riesgo para presentar la enfermedad. ${ }^{12}$

Con los recursos tanto humanos como materiales con los que cuenta esta unidad hospitalaria, cabe destacar que se pudieron usar diversas herramientas diagnósticas, las cuales fueron decisivas tanto para corroborar el diagnóstico como para el tratamiento ofertado a estas pacientes, logrando con ello óptimos resultados y mayor satisfacción en las pacientes; sin embargo, es necesario realizar mayores campañas de tamizaje en la población, por lo cual es necesario seguir trabajando en el desarrollo de protocolos para abatir estos índices de morbimortalidad y así poder brindar una atención de alta calidad, otorgando un abordaje integral, diagnóstico y tratamiento oportuno, y logrando así obtener un adecuado índice de satisfacción por parte de las pacientes y su familia.

\section{CONCLUSIÓN}

Las características demográficas de nuestra muestra son similares a la población mexicana de otros estudios. El principal tipo histológico que encontramos fue el cáncer ductal infiltrante y con mayor presencia de receptores a estrógenos. Estos datos nos ayudan a establecer futuras líneas de investigación y prevención para mejorar la calidad de atención en los pacientes con una patología de alta morbilidad y mortalidad, logrando con ello mejorar la calidad de vida.

\section{REFERENCIAS}

1. McDonald S, Saslow D, Alciati MH. Performance and reporting of clinical breast examination: a review of the literature. CA Cancer J Clin. 2004; 54 (6): 345-361.

2. Mahoney L, Csima A. Efficiency of palpation in clinical detection of breast cancer. Can Med Assoc J. 1982; 127 (8): 729-730.

3. Agarwal T, Patel B, Rajan P, Cunningham DA, Darzi A, Hadjiminas DJ. Core biopsy versus FNAC for palpable breast cancers. Is image guidance necessary? Eur J Cancer. 2003; 39 (1): 52-56.

4. Pérez-Sánchez VM, Vela-Chávez TA, Mora-Tiscareño A. Diagnóstico histopatológico y factores pronósticos en cáncer infiltrante de la glándula mamaria. Cancerología. 2008; 3: 7-17.

5. Sørlie T, Perou CM, Tibshirani R, Aas T, Geisler S, Johnsen $\mathrm{H}$ et al. Gene expression patterns of breast carcinomas distinguish tumor subclasses with clinical implications. Proc Natl Acad Sci U S A. 2001; 98 (19): 10869-10874.

6. Slodkowska EA, Ross JS. MammaPrint 70-gene signature: another milestone in personalized medical care for breast cancer patients. Expert Rev Mol Diagn. 2009; 9 (5): 417-422.

7. Albain KS, Paik S, van't Veer L. Prediction of adjuvant chemotherapy benefit in endocrine responsive, early breast cancer using multigene assays. Breast. 2009; 18 Suppl 3: S141-S145.

8. Huicochea-Castellanos S, González-Balboa P, Tovar-Calderón IL, Olarte-Casas MA, Vázquez-Lamadrid J. Cáncer de mama. Anales de Radiología México. 2009; 8 (1): 117-126.

9. Ziv E. Genética del cáncer de mama: aplicaciones para la población mexicana. Salud Pública Mex. 2011; 53 (5): 415-419.

10. Mohammed RH, Lakatua DJ, Haus E, Yasmineh WJ. Estrogen and progesterone receptors in human breast cancer. Correlation with histologic subtype and degree of differentiation. Cancer. 1986; 58 (5): 1076-1081.

11. Salinas-Martínez AM, Juárez-Ruiz A, Mathiew-Quirós A, Guzmán-De la Garza FJ, Santos-Lartigue A, Escobar-Moreno C. Cáncer de mama en México: tendencia en los últimos 10 años de la incidencia y edad al diagnóstico. Rev Invest Clin. 2014; 66 (3): 210-217.

12. Mohar A, Reynoso N, Villarreal-Garza C, Bargalló-Rocha JE, ArceSalinas C, Lara-Medina F. Cáncer de mama en el Instituto Nacional de Cancerología. Experiencia del Seguro Popular 2007-2013. Rev Mex Mastol. 2015; 5 (1): 6-11. 